\title{
REVIEW ARTICLE \\ Immunopathogenesis and Treatment of Rheumatoid Arthritis: Recent Developments
}

\author{
ASM Giasuddin ${ }^{1}$, KA Jhuma ${ }^{2}$, AMM Haq $^{3}$, MM Haque $^{3}$ \\ ${ }^{1}$ Dept of Biochemistry \& Immunology, State College of Health Sciences, Dhaka \\ ${ }^{2}$ Dept of Biochemistry, Medical College for Women \& Hospital, Dhaka \\ ${ }^{3}$ Dept of Medicine, Medical College for Women \& Hospital, Dhaka
}

\begin{abstract}
Rheumatoid arthritis (RA) is a chronic inflammatory disease occurring three times more in females throughout the world affecting $1-2 \%$ of the adult population in all ethnic groups, usually in the age group of 25-60 years. Although the role of $\mathrm{CD}_{4}{ }^{+} \mathrm{T}$ helper lymphocytes in the aetiopathogenesis has been studied for more than three decades, the focus on $\mathrm{CD}_{4}{ }^{+}$ $\mathrm{T}$ helper type 17 (Th17) lymphocytes and its associated cytokines is much more recent. The cytokines such as IL-17 and IFN- $\gamma$ induce secondary cytokines such as IL-1, TNF- $\alpha$, etc which possibly cause inflammation in joints. This cytokine cascade, therefore, offers a number of points and opportunities for immunointervention in RA. The present review article highlights some of the major aspects of the immunopathogenesis that involve Th17 cells and their association relevant to recent developments in the treatment of RA.
\end{abstract}

Key Words: Rheumatoid arthritis, Immunopathogenesis, Cytokines, Interlenkin- $17, \mathrm{CD}_{4}{ }^{+} \mathrm{Th} 17$ cell

\section{Background}

Rheumatoid arthritis (RA) is a chronic inflammatory autoimmune and systemic disease characterized by long-term chronic synovial inflammation, hyperplasia and joint damage leading to functional decline and disability as well as increased mortality. Systemic features of RA include cardiovascular complications, pulmonary complications and skeletal disorders. Although systemic manifestations may be present at the onset, they develop more usually as the disease progresses. The diagnosis of RA is made on clinical grounds mainly. However, the blood abnormalities such as the presence of rheumatoid factor (RF) is usually useful in the diagnosis of RA. RF is detected in the majority $(70-80 \%)$ of patients with established disease and constitutes one of the American College of Rheumatology (ACR) classification criteria. RA is a common disease occurring three times more in females throughout the world affecting $1-2 \%$ of the adult population in all ethemic groups, usually in the age group of $25-60$ year $^{1-7}$.

Bangladesh J Med Biochem 2014; 7(2): 62-67
Thus, it seems critical to understand the underlying pathogenetic mechanisms behind RA in order to design better treatment strategies. This review article highlights some of the major aspects of the immunopathogenesis that involve $\mathrm{CD}_{4}{ }^{+}$Th17 cells and their association relevant to recent developments in the treatment of RA.

\section{Etiopathogenesis of Rheumatoid Arthritis}

Numerous hypotheses have been proposed to unravel the mystery behind the development of RA, but none has completely illustrated the cause that underlies the slow, progressive and chronic inflammatory response in the joints that ultimately leads to joint damage and severe disability. However, RA is considered as an autoimmune disease as autoimmune process contributes to the pathogenesis of the disease. Several studies reported that $\mathrm{T}$ cells are major culprit in initiating the detrimental inflammatory response in the joints ${ }^{2,3}$. In addition, the role of the humoral immune response is seen as equally important in 
Immunopathogenesis and Treatment of Rheumatoid Arthritis: autoimmune RA, where auto-antibodies (rheumatoid factor, RF) are generated against the healthy joint tissue. Over the long term these auto-antibodies damage the tissue of the joints leading to the pathological condition in RA $\left(\right.$ Figure-1) ${ }^{8}$. Several studies have suggested that $\mathrm{RA}$ is triggered by genetic, environmental, epigenetic and dietary factors 8,9 .

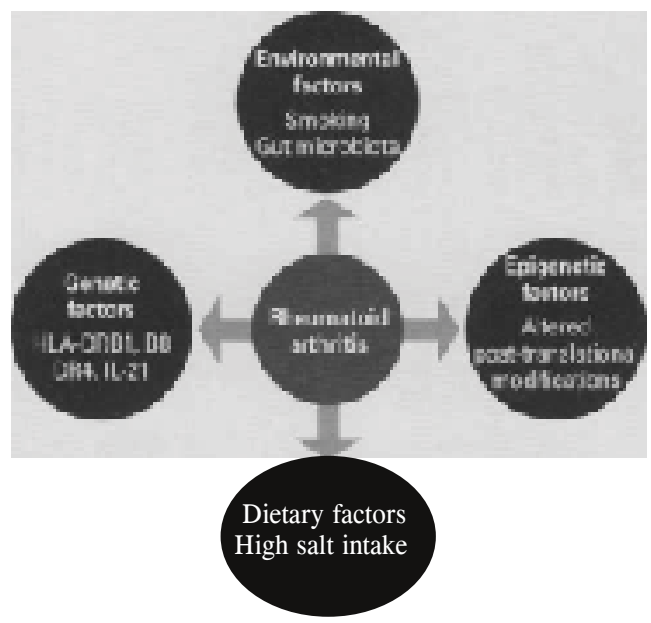

Figure-1: Pathological triggers of RA. Genetic factors such as alterations to the genes for HLA-DRB1, B8, DR4 and IL-1 IF may trigger the development of RA by generating an inflammatory response. Environmental factors including smoking and intestinal microbiota are also thought to be involved in a reduction in tolerance, which can induce autoimmunity by producing autoantibodies (RA factor). Moreover, it has also been suggested that a high salt diet might play a role in triggering the joint inflammation in RA by inducing pathogenic Th17 cells (adapted from reference 8).

The most accepted hypothesis is the inflammatory response mediated by humoral and cell mediated immune responses. The role of $\operatorname{IgG}$ autoantibodies and immune complexes has been recognized as a potent pathological trigger of inflammatory response, and autoantibodies (RF) are detected in more than $80 \%$ of RA patients. These autoantibodies consistently erode the lining of the joints, producing inflammation of the synovium and the symptoms associated with $\mathrm{RA}^{2,3,9}$. However, the major concerns in RA are associated with the inflammation triggered by $\mathrm{T}$ lymphocytes. More recently, the role of $\mathrm{CD}_{4}+\mathrm{T}$ helper lymphocytes has been established as the major contributor to chronic inflammation in RA (Figure-2) ${ }^{10,11}$.

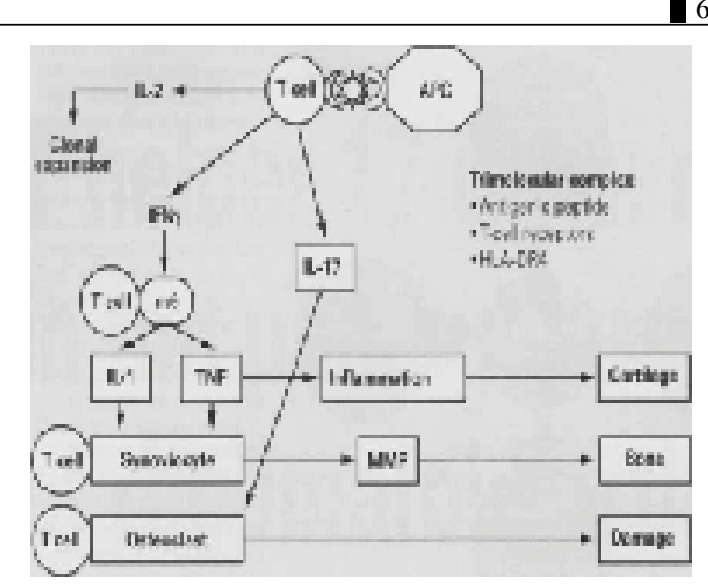

Figure-2: T- cell mediated pathway of RA. Antigen presenting cells (APC) activate $\mathrm{T}$ cells which in turn activate specific cytokines (eg IL-2, IL-17 and IFN-g). These cytokines in turn activate secondary cytokines (mainly IL-1 and TNF-a) that induce inflammation in joints (adapted from reference 11).

The role of $\mathrm{T}$ cells in the pathogenesis of RA has been studied for more than three decades. However, the focus on a particular subset of $\mathrm{CD}_{4}{ }^{+} \mathrm{T}$ helper cells, i.e. $\mathrm{T}$ helper cells type 17 (Th17) and its associated cytokines particularly $1 \mathrm{~L}-17$, is much more recent. Studies to know the role of Th17 in RA have already provided a deeper understanding of the pathophysiological mechanisms involved in triggering the detrimental inflammatory response. $1 \mathrm{~L}-17$ and $1 \mathrm{FN}-\gamma$ in turn induce production of secondary cytokines such as $1 \mathrm{~L}-1$ and TNF $\alpha$ which cause inflammation in joints ${ }^{10,11}$. However, the complete mechanism behind this complex process remains enigmatic and controversial, and requires further study both in human and animal models.

\section{T Helper 17 (Th17) Cells \& Their Cytokines}

Upon activation, $\mathrm{CD}_{4}{ }^{+} \mathrm{T}$ cells differentiate into $\mathrm{T}$ helper subsets with different cytokine profiles and functions. Until recently, $\mathrm{T}$ helper cells were categorized into two subsets, Th1 and Th2, depending on the cytokines produced. However, with progress in immunological techniques and intense research in the area, it is now apparent that there is a third subset, Th17, which produces the cytokine IL-17 12 . The physiological function of Th17 cells is to provide host defense against extracellular bacteria and some fungi against which Th1 and Th2 cells are not fully effective ${ }^{13}$. 
However, their essential role in autoimmune diseases is a major cause of concern, as numerous studies, both in animal and human experimental models, have suggested a pathological role.

Recent reports suggest that IL-17 is a proinflammatory cytokine that induces the production of other inflammatory cytokines, e.g. IL-1, IL-6, IL-8, IL-21, IL-23 and TNF- $\alpha$. These cytokines have been implicated in the pathogenesis of many inflammatory diseases including RA. Moreover, It is suggested that IL- 6 and TNF- $\alpha$ induce the differentiation of Th17 cells, whereas IL-21 and IL23 promote the proliferation of Th17 cells. Furthermore, studies reported that the cytokines produced by Th17 are co-related with the cytokines produced by another pro-inflammatory $\mathrm{T}$ helper cells subset, Th1, suggesting a central role for Th17 in the development of inflammatory disease ${ }^{14}$.

Initially it was suggested that the proinflammatory cytokine IL-23 played a prominent role in the differentiation of Th17, as IL-23deficient transgenic mice have been reported to lack IL-17-producing T cells. In vitro, however, IL-23 did not seem sufficient to generate Th17 from native T-cells precursors. Furthermore, in the presence of transforming growth factor- $\beta$ (TGF- $\beta$ ) and IL-6, the role of IL-23 in Th17 differentiation appeared to be dispensable. These findings suggested that TGF- $\beta$, along with IL-6, is both central and sufficient in Th17 differentiation ${ }^{15}$.

However, Kato and Fox reported that the combination of TGF- $\beta$ and IL- 6 does not induce Th17 cells, a finding supported by Manel et al., who also reported that TGF- $\beta$ and IL- 6 are not sufficient for the differentiation of Th17 cells in humans. Their study suggested that TGF- $\beta$, along with IL-1 and IL-6 or IL-23, is necessary for human Th17 cells differentiation. Thus, in humans, the differentiating factor for Th17 remains controversial and requires further investigation ${ }^{13,16}$.

\section{Role of Th17 Cells}

Various hypotheses have proposed a role for Th17 in RA. As mentioned previously, IL-17 has the potential to activate a diverse range of cell types that are implicated in the pathogenesis of RA.
Induction of chemokines by IL-17 indirectly attracts $\mathrm{T}$ and $\mathrm{B}$ cells, monocytes and neutrophils to the inflamed joint, all of which contribute to the pathogenesis of RA ${ }^{13}$. Kim et al. reviewed the role of Th17 and their associated cytokines in 55 RA patients and 20 osteoarthritis (OA) patients and assessed disease activity using the 28 -joint disease activity score (DAS28). Furthermore, they also evaluated erythrocyte sedimentation rate (ESR), serum C-reactive protein (CRP) and RF level, and quantified IL-6, IL-17, IL-21, IL-23 and TNF- $\alpha$ using an enzyme-linked immunosorbent assay (ELISA) technique. Statistical analysis showed that a comparison of the frequency of Th17 cells in a patient with active and inactive RA was significant $(\mathrm{P}=0.03)$, suggesting that Th17 was significantly higher in active RA patients or in OA patients $(\mathrm{P}=0.003)$. Interestingly, the findings showed no significant difference in the frequency of Th1 cells between the active RA $(\mathrm{P}=0.29)$ and $\mathrm{OA}$ $(\mathrm{P}=0.10)$ groups. Thus, it was concluded that the Th17 subset has a wider role to play in the pathogenesis of RA than does the Th1 subset. Furthermore, an association between Th17 and RA was supported by the finding of a positive correlation between the percentages of Th17 cells in RA patients, the inflammatory marker CRP and the DAS28 score (Figure-3) ${ }^{14}$. However, the study failed to explain the implications of the other two major cytokines i.e., IL-23 and TNF- $\alpha$ in RA. Overall, the findings do show some correlation between Th17 and IL-6, IL-17 and IL21, suggesting a pathological effect in RA. More importantly, the levels of these cytokines were also positively correlated with inflammatory markers such as CRP and ESR, adding further evidence for a connection between Th17 and RA ${ }^{14}$. Other clinical studies implicated Th17 cells in the pathogenesis of RA using flow cytometric analysis of cells and EIA for cytokines 17,18 . The studies found that frequencies of Th17 cells and IL-17 strongly correlated with disease activity both at the onset and during progression of RA. 
Immunopathogenesis and Treatment of Rheumatoid Arthritis:

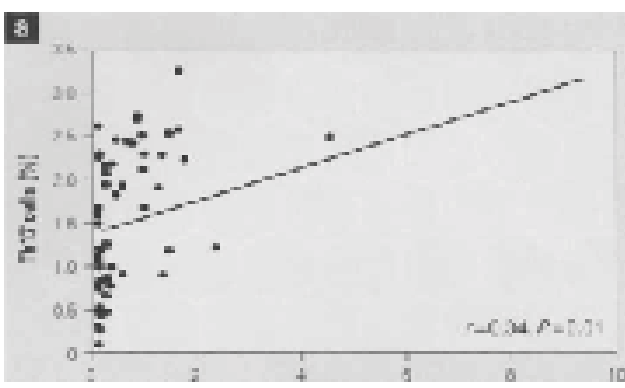

$\mathrm{C}$ - reactive protein $(\mathrm{CRP})(\mathrm{mg} / \mathrm{dl})$

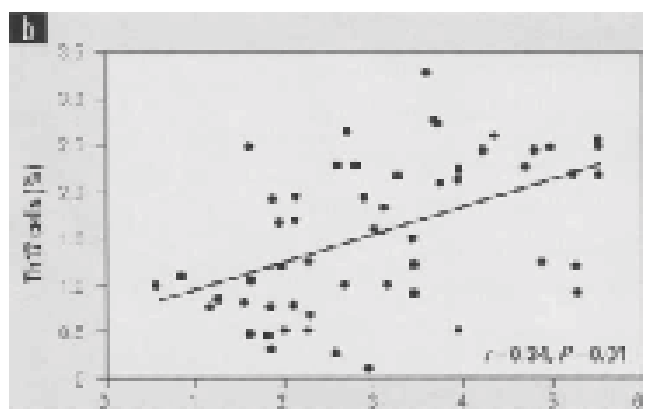

DAS 28 Score

Figure-3: Correlation between the percentage of Th17 cells and a) C-reactive protein and b) DAS28 in RA patients (adapted from reference 14)

However, the study failed to show a correlation between Th17 frequency and CRP level in patients and was limited in that it looked only at IL-17. Overall, the study established an association between Th17 cells and their cytokines, suggesting a pathological mechanism behind $\mathrm{RA}^{17}$.

\section{Recent Development in Treatment}

It has been stated in the etiopathogenesis of RA that IL-17 and IFN- $\gamma$ from $\mathrm{CD}_{4}{ }^{+}$Th cells in turn activate production of secondary cytokines mainly IL-1 and TNF- $\alpha$ from macrophage that induced inflammation in joints. This cytokines cascade therefore offers a number of points and opportunities for immunointervention ${ }^{17-20}$.

In rheumatoid arthritis (RA), the conventional therapies (first-line, second-line, third-line drugs) provide more or less effective symptomatic relief for a decade or so from the onset of the disease. The mainstay of treatment in RA comprises the early use of small molecule disease-modifying antirheumatic drugs (DMARDs) for induction of remission with or without steroids. Among the
DMARDs, the first-line drugs (non-steroidal antiinflammatory drugs, NSAIDs) are methotrexate, sulfasalazine, hydrochloroquine, leflunomide, gold, D-penicillamine, azathiorine, cyclophosphamide and cyclosporin. A partial or nonresponse to DMARDs therapy prompts increase in the dose or use of an additional DMARD with or without corticorteroids such as prednisoline, methye prednisolone (second line drugs) ${ }^{21-23}$. However, the chronic inflammatory destructive processes involving connective tissue, cartilage and bone with their attendant disability progress relentlessly in majority of patients. Secondly, use of 'second-line' and 'third-line' drugs in RA are limited due to their side effects ${ }^{21-23}$. Regarding the third-line therapy studies in animal and RA patients have confirmed that TNF- $\alpha$, an inflammatory cytokine, is of major importance in the rheumatoid disease process and thus, it might be an effective therapeutic target in RA. Animal model experiments and clinical trials were conducted with anti-TNF monoclonal antibody (anti-TNF- $\alpha$ MoAb) in RA recently. This antiTNF- $\alpha$ MoAb therapy was found to be both effective and safe which documented the comingof-age of cytokine-based immunointervention in $\mathrm{RA}^{18,20}$. However, The use of biological therapies with biologies (third-line drugs) such as anti-TNF MoAb, anti-CD20 receptor antibody therapy (Retuximab), anti-T cell activation therapy (Abatacept), anti IL-6 receptor therapy (Tocilizumab), Anti IL-1 receptor (Anaklora), etc are reserved for the treatment of patients who have high disease activity despite having an adequate trial of traditional DMARDs with or without steroids. The biologics are targeted towards specific cytokines and other cell-surface molecules regulating the immune response. Although generally well tolerated, biological therapies increase the risk of serious infections, reactivation of tuberculosis and development of malignancies due to suppression of the immune response. Although biological treatment is more effective than standard DMARD therapy, treatment costs are significantly greater. Because of this, many countries have set guidelines restricting their use. Current UK recommendations are that biological therapy should be initiated only in active RA (DAS $28>5.1$ ) when an adequate 
trial of at least two other DMARDs (including methotrexate) has failed ${ }^{23}$.

The focus on Th17 cells and their cytokines is fairly new. Different conclusions have emerged about the potential role of Th17 in RA and interesting findings implicate Th17 cells rather than the more generally accepted Th1-mediated inflammatory response in $\mathrm{RA}^{14}$. In another study, Leipe et al implicated a role for Th17 in RA both in the early and late stages and they established that Th17 cells enhance production of IL-17 which induces systemic inflammation in $\mathrm{RA}^{17}$. Similarly, Janke et al explained the pathological potential of Th17 cells in both in vitro and in vivo experimental models, suggesting that the combination of TGF- $\beta$ and IL-6 is sufficient to induce inflammatory response in models in vivo ${ }^{18}$. It is clear that Th17 cells may have different roles in different experimental models. In vitro models showed greater correlation between Th17 cytokines and RA, perhaps due to the fact that the in vitro model system lacks the microenvironment required for differentiation and proliferation of Th17 cells naturally. In the animal model, the role of Th17 cells could be different as many interactions occur with other components of the immune system, all of which may affect the differentiation and proliferation of Th17 cells ${ }^{24,25}$.

In conclusion, such findings obviously require further study to develop new immunotherapeutic interventions. This would help to achive greater success of cytokine-based immunointervention without side-effects and to develop it as the 'fourth-line' treatment modality for a wide range of autoimmune diseases including RA. Researchers are optimistic that modern medicine would certainly witness the application of this noble immunotherapy enabling to selectively target cytokines, e.g. TNF- $\alpha$, IL-17 and other cytokines in the cascade possibly in RA in the near future.

\section{References}

1. Dohertty M, Ralston SH. Musculoskeletal disease (Inflammatory joint disease). In: Colledge NR, Walker BR, Ralston SH (Editors). Davidson's Principle \& Practice of Medicine, 21st Edition; Edinburgh: Churchill Livingstone (Elsevier); 2010: 1088-1105.
2. Delves PJ, Martin SJ, Burton DR, Roitt IM. Roitt's Essential Immunology, 11th Edition; Oxford, UK: Blackwell Publishing Ltd; 2006: 410-55.

3. Chapel H, Haeney M, Misbah S, Froden N. Essentials of Clinical Immunology, 4th Editon; Oxford, UK: Blackwell Science Ltd; 1999: 183-205.

4. Abreu I, Laroche P, Bastor A, Issert V, Cruz M et al. Multiplexed immunoassay for detection of rheumatoid factors by FIDISTM technology. Ann NY Acad Sci 2005; 1050 : 357-63.

5. Voll RE, Kalden JR. Do we need new treatment that goes beyond tumor necrosis factor blockers for rheumatoid arthritis? Ann NY Acad Sci 2005; 1051: 799-810.

6. Fuchs HA, Sergent JS. Rheumatoid arthritis: the clinical picture. In: Arthritis and Allied Conditions: A Textbook of Rheumatology; Baltimore: Williams \& Wilkins; 1997: 1041-70.

7. Arnet FC, Edworthy SM, Bloch DA, McShane DJ, Fries JF, Cooper NS et al. The American Rheumatism Associaton 1987 revised criteria for classification of rheumatoid arthritis. Arthritis Rheum 1988; 31: 315-24.

8. McInnes IB, Schett G. The pathogenesis of rheumatoid arthritis. N Eng J Med 2011; 365: 2205-19.

9. Silverman GJ, Carson DA. Roles of B cells in rheumatoid arthritis. Arthritis Res Ther 2003; 5: 1-6.

10. Solomon GE. T-cell agents in the treatment of rheumatoid arthritis. Bull NYU Hosp Jt Dis 2010; 68: 162-65.

11. Panayi GS. B cells: a fundamental role in the pathogenesis of rheumatoid arthritis? Rheumatology (Oxford) 2005; 44: 23-27.

12. Korn T, Bettelli E, Oukka M, Kuchroo VK. IL-17 and Th17 cells. Annu Rev Immunol 2009; 27: 485517.

13. Kato H, Fox DA. Are Th17 cells an appropriate new target in the treatment of rheumatoid arthritis? Clin transl Sci 2010; 3: 319-26.

14. Kim J, Kang S, Kim J, Kwon G, Koo S. Elevated levels of $\mathrm{T}$ helper 17 cells are associated with disease activity in patients with rheumatoid arthritis. Ann Lab Med 2013; 33: 52-59.

15. Stockinger B, Veldhoen M. Differentiation and function of Th17 T cells. Curr Opin Immunol 2007; 19: 281-86. 
16. Manel N, Unutmaz D, Littman DR. The differentiation of human Th17 cells requires transforming growth factor - and induction of the nuclear receptor ROR T. Nat Immunol 2008; 9: 641-49.

17. Leipe J, Grunke M, Dechant C, Reihdl C, Kerzendorf U, Schulze-Koops $\mathrm{H}$ et al. Role of Th17 cells in human autoimmune arthritis. Arthritis Rheum 2010; 62: 2876-85.

18. Janke M, Peine M, Nass A, Morawietz L, Hamann A, Scheffold A et al. In vitro-induced Th17 cells fail to induce inflammation in vivo and show an impaired migration into inflammed sites. Eur $\mathbf{J}$ Immunol 2010; 40: 1089-98.

19. Giasuddin ASM, Ziu MM. Views for future development of cytokines in health and disease: implications for clinical medicine. J Islam Acad Sci 1996; 9: 67-74.

20. Giasuddin ASM, Hoque ME, Ahmed ZU. Tumour necrosis factor-alpha as a new therapeutic target for rheumatoid arthritis: An update. Bangladesh Med Res Counc Bull 2005; 31: 88-94.
21. Wordsworth BP. Rheumatoid arthritis. In: Weatherall DI, Ledingham JGG, Warell DA (Editors). Oxford Textbook of Medicine, Vol. 3, Third Edition; Oxford: Oxford University Press; 1996: 2953-65.

22. Lipsky PE. Rheumatoid arthritis. In: Fauci AS, Braunwald E, Isselbacher $\mathrm{KJ}$, et al (Editors). Harrison's Principles of Internal Medicine, Vol. 2, Fourteenth Edition; New York: McGraw-Hill; 1998: $1880-88$.

23. Ralston S H, McInnes I B. Rheumatology and bone disease. In: Walker BR, College NR. Ralston S H, Penman ID (Editors). Davidson's Principles and Practice of Medicine, 22nd Edition; Edinburgh: Churchill Livingstone / Elsevier Ltd; 2014: 1057-1135.

24. Eisenstein EM, Williams CB. The T (reg) Th17 cell balance: a new paradigm for autoimmunity. Pediatr Res 2009; 65: 26R-31R.

25. Sarkar S, Cooney LA, Fox DA. The role of $\mathrm{T}$ helper type 17 cells in inflammatory arthritis. Clin Exp Immunol 2010; 159: 225-37. 